DOI: $10.15393 / \mathrm{j} 3 . \operatorname{art} .2019 .6410$

UDC 517.521

M. S. SultanakHMEdov

\title{
ON THE CONVERGENCE OF THE LEAST SQUARE METHOD IN CASE OF NON-UNIFORM GRIDS
}

\begin{abstract}
Let $f(t)$ be a continuous on $[-1,1]$ function, which values are given at the points of arbitrary non-uniform grid $\Omega_{N}=$ $=\left\{t_{j}\right\}_{j=0}^{N-1}$, where nodes $t_{j}$ satisfy the only condition $\eta_{j} \leqslant t_{j} \leqslant \eta_{j+1}$, $0 \leqslant j \leqslant N-1$, and nodes $\eta_{j}$ are such that $-1=\eta_{0}<\eta_{1}<\eta_{2}<$ $<\cdots<\eta_{N-1}<\eta_{N}=1$. We investigate approximative properties of the finite Fourier series for $f(t)$ by algebraic polynomials $\hat{P}_{n, N}(t)$, that are orthogonal on $\Omega_{N}=\left\{t_{j}\right\}_{j=0}^{N-1}$. Lebesgue-type inequalities for the partial Fourier sums by $\hat{P}_{n, N}(t)$ are obtained.

Key words: random net, non-uniform grid, orthogonal polynomials, Legendre polynomials, least square method, Fourier series, function approximation

2010 Mathematical Subject Classification: 42C10, 41A10, $33 F 05$
\end{abstract}

1. Introduction. Let $\left\{\eta_{j}\right\}_{j=0}^{N}$ be a system of points, such that

$$
-1=\eta_{0}<\eta_{1}<\eta_{2}<\cdots<\eta_{N-1}<\eta_{N}=1 .
$$

We assume $\Delta \eta_{j}=\eta_{j+1}-\eta_{j}, 0 \leqslant j \leqslant N-1, \lambda_{N}=\max _{0 \leqslant j \leqslant N-1} \Delta \eta_{j}$. Now, we construct a grid $\Omega_{N}$ from the points

$$
\eta_{j} \leqslant t_{j} \leqslant \eta_{j+1}, \quad j=0,1, \ldots, N-1,
$$

selected on each segment $\left[\eta_{j}, \eta_{j+1}\right]$. Without loss of generality, we can consider all the nodes $\left\{t_{j}\right\}_{j=0}^{N-1}$ distinct, because if $t_{j}=t_{j+1}$ for some $j$, we can leave only one of them and denote the grid by $\Omega_{N-1}$.

Consider the space $l_{2}\left(\Omega_{N}\right)$ of discrete functions $f: \Omega_{N} \rightarrow R$, where the inner product is given by

$$
\langle f, g\rangle=\sum_{j=0}^{N-1} f\left(t_{j}\right) g\left(t_{j}\right) \Delta \eta_{j}=\lambda_{N} \sum_{j=0}^{N-1} f\left(t_{j}\right) g\left(t_{j}\right) \rho_{j} .
$$

(C) Petrozavodsk State University, 2019 
By $\hat{P}_{n, N}(t), 0 \leqslant n \leqslant N-1$, we denote polynomials that form finite orthonormal system with respect to this inner product:

$$
\left\langle\hat{P}_{n, N}, \hat{P}_{m, N}\right\rangle=\sum_{j=0}^{N-1} \hat{P}_{n, N}\left(t_{j}\right) \hat{P}_{m, N}\left(t_{j}\right) \Delta \eta_{j}= \begin{cases}0, & n \neq m, \\ 1, & n=m .\end{cases}
$$

We call polynomials $\hat{P}_{n, N}(t), 0 \leqslant n \leqslant N-1$, the discrete orthonormal Legendre polynomials.

Since the system $\left\{\hat{P}_{n, N}(t)\right\}_{n=0}^{N-1}$ is complete in $l_{2}\left(\Omega_{N}\right)$, any function $f \in l_{2}\left(\Omega_{N}\right)$ can be expanded in a finite Fourier series by this system. Let $\Lambda_{n, N}(f, t)$ be the partial Fourier sum of order $n$ for the function $f=f(t)$ by the system $\left\{\hat{P}_{k, N}\right\}_{k=0}^{N-1}$, in other words

$$
\Lambda_{n, N}(f, t)=\sum_{k=0}^{n} \hat{f}_{k} \hat{P}_{k, N}(t), \quad \text { where } \quad \hat{f}_{k}=\sum_{j=0}^{N-1} f\left(t_{j}\right) \hat{P}_{k, N}\left(t_{j}\right) \Delta \eta_{j} .
$$

The main goal of this article is to study the approximative properties of $\Lambda_{n, N}(f, t)$ in case when $f(t)$ is continuous on $[-1,1]$ and $t \in[-1,1]$. More precisely, we want to obtain an estimate for the value

$$
\left|R_{n, N}(f, t)\right|=\left|f(t)-\Lambda_{n, N}(f, t)\right|, \quad t \in[-1,1] .
$$

Note that the value $\left|R_{n, N}(f, t)\right|$ for the discrete Legendre polynomials was studied in [2] for the case of $t_{j}=\eta_{j}$ and was studied in [3] for the case of $t_{j}=\frac{\eta_{j}+\eta_{j+1}}{2}$. But the results obtained there are valid only when $n=O\left(\lambda_{N}^{-1 / 5}\right)$ and $n=O\left(\lambda_{N}^{-2 / 7}\right)$, respectively, while we managed to get estimates for $n=O\left(\lambda_{N}^{-1 / 3}\right)$ and for a more general case when $t_{j}$ is arbitrary on the segment $\left[\eta_{j}, \eta_{j+1}\right]$.

To solve this problem, we need some information about discrete Legendre polynomials $\hat{P}_{k, N}(t)$, as well as discrete Jacobi polynomials $\hat{P}_{k, N}^{\alpha, \beta}(t)$, which are a generalization of $\hat{P}_{k, N}(t)$. This information is based on the properties of classical continuous Legendre and Jacobi polynomials.

2. Some information about Jacobi and Legendre polynomials. The Jacobi polynomials can be written using Rodrigues' formula (see, for example, [4]) as follows:

$$
P_{n}^{\alpha, \beta}(t)=\frac{(-1)^{n}}{2^{n} n !} \frac{1}{\kappa^{\alpha, \beta}(t)} \frac{d^{n}}{d t^{n}}\left\{\kappa^{\alpha, \beta}(t) \sigma^{n}(t)\right\},
$$


where $\alpha, \beta$ are arbitrary real numbers, $\kappa^{\alpha, \beta}(t)=(1-t)^{\alpha}(1+t)^{\beta}, \sigma(t)=$ $=1-t^{2}$. In the case when $\alpha, \beta>-1$, the Jacobi polynomials form an orthogonal system with the weight $\kappa^{\alpha, \beta}(t)$ :

$$
\int_{-1}^{1} P_{n}^{\alpha, \beta}(t) P_{m}^{\alpha, \beta}(t) \kappa^{\alpha, \beta}(t) d t=h_{n}^{\alpha, \beta} \delta_{n m}
$$

where

$$
h_{n}^{\alpha, \beta}=\frac{2^{\alpha+\beta+1} \Gamma(n+\alpha+1) \Gamma(n+\beta+1)}{n !(2 n+\alpha+\beta+1) \Gamma(n+\alpha+\beta+1)},
$$

and, therefore, $h_{n}^{\alpha, \beta} \asymp n^{-1}, n=1,2, \ldots$ For the derivative of $P_{n}^{\alpha, \beta}(t)$, the following equality holds:

$$
\left(P_{n}^{\alpha, \beta}(t)\right)^{\prime}=\frac{\alpha+\beta+n+1}{2} P_{n-1}^{\alpha+1, \beta+1}(t) .
$$

We will also need the following weighted estimate

$$
\sqrt{n}\left|P_{n}^{\alpha, \beta}(t)\right| \leqslant c(\alpha, \beta)\left(\sqrt{1-t}+\frac{1}{n}\right)^{-\alpha-\frac{1}{2}}\left(\sqrt{1+t}+\frac{1}{n}\right)^{-\beta-\frac{1}{2}},
$$

where $-1 \leqslant t \leqslant 1$. An important particular case of Jacobi polynomials with $\alpha=\beta=0$ is Legendre polynomials $P_{n}(t)$, orthogonal on $[-1,1]$ with the unit weight $\rho(t) \equiv 1$. Denote by $\hat{P}_{n}(t)=\sqrt{\frac{2 n+1}{2}} P_{n}(t), n=0,1,2, \ldots$ the corresponding orthonormal Legendre polynomials. The leading coefficient of polynomial $\hat{P}_{n}(t)$ can be written as

$$
k_{n}=\frac{(2 n) !}{(n !)^{2} 2^{n}} \sqrt{\frac{2 n+1}{2}} .
$$

3. Discrete Jacobi and Legendre polynomials. We will use the integral analogue of the Markov inequality for estimating the derivative of an algebraic polynomial (see [5,6]), which for $r=1$ has the following form:

$$
\int_{-1}^{1}\left|q_{m}^{\prime}(t)\right| d t \leqslant c(m) m^{2} \int_{-1}^{1}\left|q_{m}(t)\right| d t
$$


where $q_{m}(t)$ is an arbitrary algebraic polynomial of degree $m$. For every $m$, denote by $\chi_{m}$ the minimum of constants $c(m)$ that satisfy inequality (9), i.e.,

$$
\chi_{m}=\sup _{q_{m}} \frac{\int_{-1}^{1}\left|q_{m}^{\prime}(t)\right| d t}{m^{2} \int_{-1}^{1}\left|q_{m}(t)\right| d t},
$$

where the upper bound is taken by polynomials $q_{m}(t)$ of degree at most $m$ and not equal to zero identically. In work [5] by N. K. Bari, it is shown that $\chi=\sup _{m \geqslant 1} \chi_{m}<\infty$. Given this fact, we derive from (9):

$$
\int_{-1}^{1}\left|q_{m}^{\prime}(t)\right| d t \leqslant \chi m^{2} \int_{-1}^{1}\left|q_{m}(t)\right| d t
$$

Let $\left\{\hat{P}_{n, N}^{\alpha, \beta}(t)\right\}_{n=0}^{N-1}$ be polynomials that form a finite orthonormal system with respect to the inner product

$$
\left\langle\hat{P}_{n, N}^{\alpha, \beta}, \hat{P}_{m, N}^{\alpha, \beta}\right\rangle=\sum_{j=0}^{N-1} \hat{P}_{n, N}^{\alpha, \beta}\left(t_{j}\right) \hat{P}_{m, N}^{\alpha, \beta}\left(t_{j}\right) \kappa^{\alpha, \beta}\left(t_{j}\right) \Delta \eta_{j}= \begin{cases}0, & n \neq m \\ 1, & n=m .\end{cases}
$$

We call these polynomials discrete orthonormal Jacobi polynomials.

In the case when the grid $\Omega_{N}$ consists of equidistant nodes $t_{j}=-1+\frac{2 j}{N-1}$, the asymptotic properties and weighted estimates for the polynomials orthogonal on $\Omega_{N}$ were first studied in the papers by I. I. Sharapudinov (see [7]). Later, I. I. Sharapudinov [8-10] and A. A. Nurmagomedov [11], [12] studied the asymptotic properties of polynomials that are orthogonal on nonuniform grids of the real axis. In particular, in [12] the author investigated the asymptotic properties of the discrete Jacobi polynomials $\hat{P}_{n, N}^{\alpha, \beta}(t)$ ( $\alpha$ and $\beta$ are integers), orthogonal on nonuniform grid $\Omega_{N}$ with $t_{j}=\frac{\eta_{j}+\eta_{j+1}}{2}, 0 \leqslant j \leqslant N-1$.

In our work [13], we investigated asymptotic properties of these polynomials in the general case of random $t_{j}$ ( $\alpha, \beta$ are still integers). When $n=O\left(\lambda_{N}^{-\frac{1}{3}}\right)$ and $n, N \rightarrow \infty$ we obtained asymptotic formula

$$
\hat{P}_{n, N}^{\alpha, \beta}(t)=\hat{P}_{n}^{\alpha, \beta}(t)+v_{n, N}^{\alpha, \beta}(t),
$$


here $\hat{P}_{n}^{\alpha, \beta}(t)$ is a normed Jacobi polynomial, and $v_{n, N}^{\alpha, \beta}(t)$ is the remainder, for which the following estimate is established:

$$
\leqslant c(\alpha, \beta, \gamma)\left(\frac{3-\lambda_{N} \chi(2 n+\alpha+\beta)^{2}}{1-\lambda_{N}^{2} \chi^{2}(2 n+\alpha+\beta)^{4}}\right)^{\frac{1}{2}}\left\{\begin{aligned}
\theta^{-\alpha-\frac{1}{2}} n^{\frac{3}{2}} \sqrt{\lambda_{N}}, & \gamma n^{-1} \leqslant \theta \leqslant \frac{\pi}{2}, \\
n^{\alpha+2} \sqrt{\lambda_{N}}, & 0 \leqslant \theta \leqslant \gamma n^{-1},
\end{aligned}\right.
$$

where $\chi$ is the smallest of the constants in the Markov integral inequality for estimating the derivative of an algebraic polynomial. Here and further in the text, $c, c(\alpha), c(\alpha, \beta), c(\alpha, \beta, \ldots, \gamma)$ are positive constants depending only on the specified parameters, which, generally speaking, may be different in different places. For the sake of simplicity, these estimates are given for the segment $[0,1]$; they apply to $[-1,0]$ in the similar way.

In the article, the indicated asymptotic formula is directly used to study the value $\left|R_{n, N}(f, t)\right|$.

4. Auxiliary statements. In this section, we collect some of the statements that will be needed in the future.

Lemma 1. Let $f(t)$ be a function, absolutely continuous on $[-1,1]$; $\left\{\eta_{j}\right\}_{j=0}^{N}$ and $\left\{t_{j}\right\}_{j=0}^{N-1}$ be systems of nodes that satisfy (1) and (2), respectively. Then

$$
\int_{a}^{b} f(t) d t=\sum_{a \leqslant t_{j} \leqslant b} f\left(t_{j}\right) \Delta \eta_{j}+r_{N}(f)
$$

for every segment $[a, b] \subset[-1,1]$, where

$$
\left|r_{N}(f)\right| \leqslant \lambda_{N} \int_{a}^{b}\left|f^{\prime}(t)\right| d t .
$$

Proof of this lemma can be found in [13].

From Lemma 1 the next statement also follows:

Lemma 2. Let $\left\{\eta_{j}\right\}_{j=0}^{N}$ and $\left\{t_{j}\right\}_{j=0}^{N-1}$ be systems of nodes that satisfy (1) and (2), respectively. Then the following inequality holds for an absolutely continuous on $[-1,1]$ monotonous non-negative function $f(x)$ :

$$
\sum_{a \leqslant t_{j} \leqslant b} f\left(t_{j}\right) \Delta \eta_{j} \leqslant \int_{a}^{b} f(t) d t+\lambda_{N}|f(b)-f(a)| .
$$


Lemma 3. For the leading coefficients of the discrete Legendre polynomials, the inequality

$$
\frac{k_{n, N}}{k_{n+1, N}} \leqslant 1
$$

holds; here $k_{n, N}$ and $k_{n+1, N}$ are the leading coefficients of the polynomials $\hat{P}_{n, N}$ and $\hat{P}_{n+1, N}$, respectively.

Proof. Following [14], let us consider the expression

$$
\begin{gathered}
\sum_{j=0}^{N-1} \hat{P}_{n+1, N}\left(t_{j}\right) t_{j} \hat{P}_{n, N}\left(t_{j}\right) \Delta \eta_{j}= \\
=\frac{k_{n, N}}{k_{n+1, N}} \sum_{j=0}^{N-1} \hat{P}_{n+1, N}^{2}\left(t_{j}\right)+\sum_{j=0}^{N-1} \hat{Q}_{n, N}\left(t_{j}\right)=\frac{k_{n, N}}{k_{n+1, N}} .
\end{gathered}
$$

On the other hand,

$$
\begin{aligned}
& \frac{k_{n, N}}{k_{n+1, N}} \leqslant \sum_{j=0}^{N-1}\left|\hat{P}_{n+1, N}\left(t_{j}\right)\right|\left|t_{j} \hat{P}_{n, N}\left(t_{j}\right)\right| \Delta \eta_{j} \leqslant \\
& \leqslant \max _{0 \leqslant j \leqslant N-1}\left\{\left|t_{j}\right|\right\} \sum_{j=0}^{N-1}\left|\hat{P}_{n+1, N}\left(t_{j}\right)\right|\left|\hat{P}_{n, N}\left(t_{j}\right)\right| \Delta \eta_{j} .
\end{aligned}
$$

Applying the Cauchy-Bunyakovsky inequality, we finally get

$$
\begin{gathered}
\frac{k_{n, N}}{k_{n+1, N}} \leqslant \max _{0 \leqslant j \leqslant N-1}\left\{\left|t_{j}\right|\right\}\left(\sum_{j=0}^{N-1}\left|\hat{P}_{n+1, N}\left(t_{j}\right)\right| \Delta \eta_{j}\right)^{\frac{1}{2}} \times \\
\times\left(\sum_{j=0}^{N-1}\left|\hat{P}_{n, N}\left(t_{j}\right)\right| \Delta \eta_{j}\right)^{\frac{1}{2}}=\max _{0 \leqslant j \leqslant N-1}\left\{\left|t_{j}\right|\right\} \leqslant 1 .
\end{gathered}
$$

This completes the proof.

The following lemma establishes the relation between polynomials of degrees $n$ and $n+1$.

Lemma 4. For $A_{n}=\sqrt{\frac{k_{n, N}}{k_{n+1, N}}}$ the following equalities hold: 


$$
\begin{aligned}
& (1-t) \hat{P}_{n, N}^{1,0}(t)= \\
& =A_{n}\left(\hat{P}_{n, N}(t) \sqrt{\frac{\hat{P}_{n+1, N}(1)}{\hat{P}_{n, N}(1)}}-\hat{P}_{n+1, N}(t) \sqrt{\frac{\hat{P}_{n, N}(1)}{\hat{P}_{n+1, N}(1)}}\right), \\
& (1+t) \hat{P}_{n, N}^{0,1}(t)= \\
& =A_{n}\left(\hat{P}_{n, N}(t) \sqrt{\frac{-\hat{P}_{n+1, N}(-1)}{\hat{P}_{n, N}(-1)}}-\hat{P}_{n+1, N}(t) \sqrt{\frac{-\hat{P}_{n, N}(-1)}{\hat{P}_{n+1, N}(-1)}}\right) .
\end{aligned}
$$

Proof. Consider the polynomial $Q_{n}(t)$, given by the equality

$$
(1-t) Q_{n}(t)=\hat{P}_{n+1, N}(1) \hat{P}_{n, N}(t)-\hat{P}_{n, N}(1) \hat{P}_{n+1, N}(t) .
$$

From its definition, we have

$$
\sum_{j=0}^{N-1} Q_{n}\left(t_{j}\right) \hat{P}_{k, N}\left(t_{j}\right)\left(1-t_{j}\right) \Delta \eta_{j}=0, \quad 0 \leqslant k \leqslant n-1 .
$$

Let $M_{l}(t)$ be an arbitrary polynomial of degree $l \leqslant n-1$. Since each polynomial $P_{k, N}(t)$ has degree $k$, it is obvious that $M_{l}(t)$ can be represented as their linear combination:

$$
M_{l}(t)=\sum_{k=0}^{l} d_{k} \hat{P}_{k, N}(t) .
$$

Then, from (15) we get

$$
\sum_{j=0}^{N-1} Q_{n}\left(t_{j}\right) M_{l}\left(t_{j}\right)\left(1-t_{j}\right) \Delta \eta_{j}=0
$$

i. e., polynomials $Q_{0}(t), \ldots, Q_{N-1}(t)$ form an orthogonal system with the weight $\kappa^{1,0}(t)=1-t$ on the grid $\Omega_{N}$. Hence,

$$
Q_{n}(t)=\gamma_{n} \hat{P}_{n, N}^{1,0}(t), \quad \gamma_{n}>0
$$


To find $\gamma_{n}$, taking into account (14), consider the expression

$$
\begin{gathered}
H_{n}=\sum_{j=0}^{N-1} Q_{n}^{2}\left(t_{j}\right)\left(1-t_{j}\right) \Delta \eta_{j}= \\
=\hat{P}_{n+1, N}(1) \sum_{j=0}^{N-1} \hat{P}_{n, N}\left(t_{j}\right) Q_{n}\left(t_{j}\right) \Delta \eta_{j}=\hat{P}_{n+1, N}(1) \frac{\tilde{k}_{n}}{\hat{k}_{n, N}},
\end{gathered}
$$

where $\tilde{k}_{n}, \hat{k}_{n, N}$ are the leading coefficients of polynomials $Q_{n}(t)$ and $\hat{P}_{n, N}(t)$, respectively.

In addition, notice that $\tilde{k}_{n}=\hat{P}_{n, N}(1) \hat{k}_{n+1, N}$ from (14), and, therefore

$$
H_{n}=\hat{P}_{n+1, N}(1) \hat{P}_{n, N}(1) \frac{\hat{k}_{n+1, N}}{\hat{k}_{n, N}} .
$$

On the other hand, we get, from (16) and (17),

$$
H_{n}=\gamma_{n}^{2} \sum_{j=0}^{N-1}\left(\hat{P}_{n, N}^{1,0}\left(t_{j}\right)\right)^{2}\left(1-t_{j}\right) \Delta \eta_{j}=\gamma_{n}^{2} .
$$

Comparing (19) with (20), we derive

$$
\gamma_{n}=\sqrt{\hat{P}_{n+1, N}(1) \hat{P}_{n, N}(1) \frac{\hat{k}_{n+1, N}}{\hat{k}_{n, N}}} .
$$

Returning to equality (14) and using (16), we have

$$
(1-t) \gamma_{n} \hat{P}_{n, N}^{1,0}(t)=\hat{P}_{n+1, N}(1) \hat{P}_{n, N}(t)-\hat{P}_{n, N}(1) \hat{P}_{n+1, N}(t) .
$$

This equality, together with (21), gives us (12).

Similarly, we derive equality (13).

Next, let us agree on the following notation:

$$
K_{n, N}(x, y)=\sum_{k=0}^{n} \hat{P}_{k, N}(x) \hat{P}_{k, N}(y) .
$$

Then, using the Christoffel-Darboux formula and Lemma 4, we can also prove the following assertion. 
Lemma 5. The following equality holds:

$$
\begin{aligned}
& K_{n, N}(x, y)= \\
& =C_{n, N}\left[\frac{1-x}{y-x} \hat{P}_{n, N}^{1,0}(x) \hat{P}_{n, N}(y)-\frac{1-y}{y-x} \hat{P}_{n, N}^{1,0}(y) \hat{P}_{n, N}(x)\right],
\end{aligned}
$$

where

$$
C_{n, N}=\sqrt{\frac{\hat{k}_{n, N} \hat{P}_{n+1, N}(1)}{\hat{k}_{n+1, N} \hat{P}_{n, N}(1)}} .
$$

The weighted estimate for the discrete Legendre polynomials, obtained by the author in [13], takes the following form:

Theorem A. Let us put $4 \lambda_{N} \chi n^{2}<1$; then there is a constant $a>0$, such that

$$
\begin{gathered}
\left|\hat{P}_{n, N}(t)\right| \leqslant c(a)\left(1+B \sqrt{n^{3} \lambda_{N}}\right)\left(\sqrt{1-t^{2}}+\frac{1}{n}\right)^{-\frac{1}{2}} \leqslant \\
\leqslant c(a)\left(1+B \sqrt{n^{3} \lambda_{N}}\right)\left\{\begin{aligned}
n^{\frac{1}{2}}, & -1 \leqslant t \leqslant-1+a n^{-2}, \\
(1+t)^{-\frac{1}{4}}, & -1+a n^{-2} \leqslant t \leqslant 0, \\
(1-t)^{-\frac{1}{4}}, & 0 \leqslant t \leqslant 1-a n^{-2}, \\
n^{\frac{1}{2}}, & 1-a n^{-2} \leqslant t \leqslant 1,
\end{aligned}\right.
\end{gathered}
$$

where

$$
B=\left(\frac{3-4 \lambda_{N} \chi n^{2}}{1-16 \lambda_{N}^{2} \chi^{2} n^{4}}\right)^{\frac{1}{2}}
$$

5. Approximative properties of the Fourier sums by $\hat{P}_{n, N}^{\alpha, \beta}(t)$. Suppose we are given the values of some continuous on $[-1,1]$ function $f(t)$ at the points of the grid $\Omega_{N}$. Our main goal is to estimate the value

$$
\left|R_{n, N}(f, t)\right|=\left|f(t)-\Lambda_{n, N}(f, t)\right|, \quad t \in[-1,1] .
$$

Denote by $\mathcal{P}_{n}$ the Hilbert space of all polynomials of degree $n$ and by

$$
E_{n}(f)=\min _{p_{n} \in \mathcal{P}_{n}} \max _{t \in[-1,1]}\left|f(t)-p_{n}(t)\right|
$$

the best approximation for the function $f(t)$ by polynomials of degree at most $n$. 
It is easy to show that $\Lambda_{n, N}\left(p_{n}, t\right)=p_{n}(t)$ for any polynomial $p_{n} \in \mathcal{P}_{n}$. Hence, using the Lebesgue-type inequality, we get

$$
\left|R_{n, N}(f, t)\right|=\left|f(t)-\Lambda_{n, N}(f, t)\right| \leqslant E_{n}(f)\left[1+L_{n, N}(t)\right],
$$

where

$$
L_{n, N}(t)=\sum_{j=0}^{N-1}\left|K_{n, N}\left(t_{j}, t\right)\right| \Delta \eta_{j}
$$

is the Lebesgue function for $\left\{\hat{P}_{n, N}\right\}_{n=0}^{N-1}$ and $K_{n, N}\left(t_{j}, t\right)$ is the kernel from (22).

Thus, it is necessary to study the Lebesgue function $L_{n, N}(t)$.

Theorem 1. There exists a real number $\gamma>0$, such that for $2 \leqslant n \leqslant$ $\leqslant \gamma \lambda_{N}^{-1 / 3}$ and $0<\varepsilon<1$ the following estimates hold:

$$
\begin{gathered}
\max _{-1 \leqslant t \leqslant 1} L_{n, N}(t) \leqslant c(\gamma) n^{\frac{1}{2}}, \\
L_{n, N}(t) \leqslant c(\gamma) \ln n, \quad-1+\varepsilon \leqslant t \leqslant 1-\varepsilon .
\end{gathered}
$$

Proof. We consider only the case $t \in[0,1]$, because for $t \in[-1,0]$ the proof is quite similar.

Let us start with $t \in\left[0,1-4 n^{-2}\right]$. We divide the sum on the right-hand side of (26) according to the following scheme:

$$
\begin{gathered}
L_{n, N}(t)=\left[\sum_{-1 \leqslant t_{j} \leqslant-\frac{1}{2}}+\sum_{-\frac{1}{2} \leqslant t_{j} \leqslant q_{1}}+\sum_{q_{1} \leqslant t_{j} \leqslant q_{2}}+\sum_{q_{2} \leqslant t_{j} \leqslant 1}\right]\left|K_{n, N}\left(t_{j}, t\right)\right| \Delta \eta_{j} \\
=A_{1}+A_{2}+A_{3}+A_{4},
\end{gathered}
$$

where $q_{1}=t-\frac{\sqrt{1-t^{2}}}{n}, q_{2}=t+\frac{\sqrt{1-t^{2}}}{n}$.

1. To estimate $A_{1}$, we use the Christoffel-Darboux formula and Lemma 3 , as well as the fact that $\left|t-t_{j}\right| \geqslant \frac{1}{2}$ for $t \in\left[0,1-4 n^{-2}\right]$ and $t_{j} \in\left[-1,-\frac{1}{2}\right]$. We have

$$
A_{1} \leqslant 2 \sum_{-1 \leqslant t_{j} \leqslant-\frac{1}{2}}\left(\left|\hat{P}_{n+1, N}(t) \hat{P}_{n, N}\left(t_{j}\right)\right|+\left|\hat{P}_{n, N}(t) \hat{P}_{n+1, N}\left(t_{j}\right)\right|\right) \Delta \eta_{j} .
$$

Again, we divide the sum into two parts: denote by $A_{11}$ the sum over $-1 \leqslant t_{j} \leqslant-1+4 n^{-2}$, and by $A_{12}$ that over $-1+4 n^{-2} \leqslant t_{j} \leqslant-\frac{1}{2}$. 
Using the weighted estimate (24) and Lemma 2, we obtain

$$
A_{11} \leqslant c n^{-1}, \quad A_{12} \leqslant c(1-t)^{-\frac{1}{4}},
$$

which means that

$$
A_{1} \leqslant c(1-t)^{-\frac{1}{4}}
$$

2. We proceed to the estimation of $A_{2}$, for which we again use the Christoffel-Darboux formula and the transformation (23) from Lemma 5. We have

$$
\begin{gathered}
A_{2} \leqslant C_{n, N}\left[\sum_{-\frac{1}{2} \leqslant t_{j} \leqslant q_{1}} \frac{1-t_{j}}{t-t_{j}}\left|\hat{P}_{n, N}^{1,0}\left(t_{j}\right) \hat{P}_{n, N}(t)\right| \Delta \eta_{j}+\right. \\
\left.+\sum_{-\frac{1}{2} \leqslant t_{j} \leqslant q_{1}} \frac{1-t}{t-t_{j}}\left|\hat{P}_{n, N}^{1,0}(t) \hat{P}_{n, N}\left(t_{j}\right)\right| \Delta \eta_{j}\right]=C_{n, N}\left[A_{21}+A_{22}\right] .
\end{gathered}
$$

Consider, firstly, $A_{21}$ :

$$
A_{21} \leqslant c(1-t)^{-\frac{1}{4}} \sum_{-\frac{1}{2} \leqslant t_{j} \leqslant q_{1}} \frac{1-t_{j}}{t-t_{j}}\left|\hat{P}_{n, N}^{1,0}\left(t_{j}\right)\right| \Delta \eta_{j}=c(1-t)^{-\frac{1}{4}} A_{211},
$$

where

$$
\begin{gathered}
A_{211}=\left[\sum_{-\frac{1}{2} \leqslant t_{j} \leqslant 0}+\sum_{0 \leqslant t_{j} \leqslant q_{1}}\right] \frac{1-t_{j}}{t-t_{j}}\left|\hat{P}_{n, N}^{1,0}\left(t_{j}\right)\right| \Delta \eta_{j} \leqslant \\
\leqslant c\left[\sum_{-\frac{1}{2} \leqslant t_{j} \leqslant 0} \frac{1-t_{j}}{t-t_{j}} \Delta \eta_{j}+\sum_{0 \leqslant t_{j} \leqslant q_{1}} \frac{\left(1-t_{j}\right)^{\frac{1}{4}}}{t-t_{j}} \Delta \eta_{j}\right] \leqslant \\
\leqslant c \sum_{-\frac{1}{2} \leqslant t_{j} \leqslant q_{1}} \frac{\left(1-t_{j}\right)^{\frac{1}{4}}}{t-t_{j}} \Delta \eta_{j} .
\end{gathered}
$$

Due to the obvious inequality $\left(1-t_{j}\right)^{\frac{1}{4}} \leqslant(1-t)^{\frac{1}{4}}+\left(t-t_{j}\right)^{\frac{1}{4}}$, we can rewrite

$$
\begin{aligned}
& A_{21} \leqslant c(1-t)^{-\frac{1}{4}} \sum_{-\frac{1}{2} \leqslant t_{j} \leqslant q_{1}}\left[\frac{(1-t)^{\frac{1}{4}}}{t-t_{j}}+\frac{\left(t-t_{j}\right)^{\frac{1}{4}}}{t-t_{j}}\right] \Delta \eta_{j}= \\
& =c\left[\sum_{-\frac{1}{2} \leqslant t_{j} \leqslant q_{1}} \frac{\Delta \eta_{j}}{t-t_{j}}+(1-t)^{-\frac{1}{4}} \sum_{-\frac{1}{2} \leqslant t_{j} \leqslant q_{1}} \frac{\Delta \eta_{j}}{\left(t-t_{j}\right)^{\frac{3}{4}}}\right]=
\end{aligned}
$$




$$
=c\left[A_{21}^{(1)}+(1-t)^{-\frac{1}{4}} A_{21}^{(2)}\right] .
$$

Using Lemma 2, the theorem condition of $\lambda_{N} n^{3} \leqslant \gamma^{3}$ and the fact that $\left(1-t^{2}\right)^{-\frac{1}{2}} \leqslant n$ at $t \in\left[0,1-4 n^{-2}\right]$, we get the estimates

$$
A_{21}^{(1)} \leqslant c \ln n, \quad A_{21}^{(2)} \leqslant c,
$$

wherefrom

$$
A_{21} \leqslant c\left(\ln n+(1-t)^{-\frac{1}{4}}\right) .
$$

We proceed to studying $A_{22}$. Applying the weighted estimate for $\hat{P}_{n, N}^{1,0}(t)$, we derive

$$
A_{22} \leqslant c(1-t)^{\frac{1}{4}} \sum_{-\frac{1}{2} \leqslant t_{j} \leqslant q_{1}} \frac{\left|\hat{P}_{n, N}\left(t_{j}\right)\right|}{t-t_{j}} \Delta \eta_{j} .
$$

Before applying the weighted estimate for $\hat{P}_{n, N}\left(t_{j}\right)$, note that $\left(1+t_{j}\right)^{-\frac{1}{4}} \leqslant$ $\leqslant 3^{\frac{1}{4}}\left(1-t_{j}\right)^{-\frac{1}{4}}$ and $\left(1-t_{j}\right)^{-\frac{1}{4}} \leqslant(1-t)^{-\frac{1}{4}}$ for $-\frac{1}{2} \leqslant t_{j} \leqslant 0$. Then

$$
\begin{aligned}
A_{22} \leqslant c(1-t)^{\frac{1}{4}} \sum_{-\frac{1}{2} \leqslant t_{j} \leqslant q_{1}} \frac{\left(1-t_{j}\right)^{-\frac{1}{4}}}{t-t_{j}} \Delta \eta_{j} \leqslant \\
\leqslant c \sum_{-\frac{1}{2} \leqslant t_{j} \leqslant q_{1}} \frac{\Delta \eta_{j}}{t-t_{j}} \leqslant c(a) \ln n .
\end{aligned}
$$

Substituting (30)-(31) in (29), we finally get

$$
A_{2} \leqslant C_{n, N}\left[A_{21}+A_{22}\right] \leqslant c\left((1-t)^{-\frac{1}{4}}+\ln n\right) .
$$

3. To estimate $A_{3}$, we do not apply any transformations, but substitute the weighted estimates directly in (26):

$$
\begin{aligned}
A_{3} & \leqslant c \frac{(n+1)}{(1-t)^{\frac{1}{4}}} \sum_{q_{1} \leqslant t_{j} \leqslant q_{2}} \frac{\Delta \eta_{j}}{\left(1-t_{j}\right)^{\frac{1}{4}}} \leqslant \\
& \leqslant c \frac{(n+1)}{(1-t)^{\frac{1}{4}}} \frac{q_{2}-q_{1}}{\left(1-q_{2}\right)^{\frac{1}{4}}} \leqslant c(a) .
\end{aligned}
$$


4. The study of the last part of the sum is similar to the study of $A_{2}$ :

$$
\begin{gathered}
A_{4} \leqslant C_{n, N}\left[\sum_{q_{2} \leqslant t_{j} \leqslant 1} \frac{1-t_{j}}{t_{j}-t}\left|\hat{P}_{n, N}^{1,0}\left(t_{j}\right) \hat{P}_{n, N}(t)\right| \Delta \eta_{j}+\right. \\
\left.+\sum_{q_{2} \leqslant t_{j} \leqslant 1} \frac{1-t}{t_{j}-t}\left|\hat{P}_{n, N}^{1,0}(t) \hat{P}_{n, N}\left(t_{j}\right)\right| \Delta \eta_{j}\right]=C_{n, N}\left[A_{41}+A_{42}\right] .
\end{gathered}
$$

In turn, $A_{41}$ can also be represented in the form of several sums

$$
\begin{aligned}
& A_{41} \leqslant c(1-t)^{-\frac{1}{4}} \times \\
& \qquad\left[\sum_{q_{2} \leqslant t_{j} \leqslant \frac{1+t}{2}}+\sum_{\frac{1+t}{2} \leqslant t_{j} \leqslant 1-n^{-2}}+\sum_{1-n^{-2} \leqslant t_{j} \leqslant 1}\right] \frac{1-t_{j}}{t_{j}-t}\left|\hat{P}_{n, N}^{1,0}\left(t_{j}\right)\right| \Delta \eta_{j}= \\
& =c(1-t)^{-\frac{1}{4}}\left[A_{41}^{(1)}+A_{41}^{(2)}+A_{41}^{(3)}\right] .
\end{aligned}
$$

Using Lemma 2 and the weighted estimates, we can obtain the following estimates for these terms:

$$
\begin{gathered}
A_{41}^{(1)} \leqslant c\left[(1-t)^{\frac{1}{4}} A_{411}^{(1)}+A_{412}^{(1)}\right]=c\left[(1-t)^{\frac{1}{4}} \ln n+1\right], \\
A_{41}^{(2)} \leqslant c n^{-\frac{1}{2}}, \quad A_{41}^{(3)} \leqslant c n^{-\frac{1}{2}} .
\end{gathered}
$$

Returning to inequality (35) and using (36)-(37), we derive

$$
A_{41} \leqslant c\left[\ln n+(1-t)^{-\frac{1}{4}}\left(n^{-\frac{1}{2}}+1\right)\right] \leqslant c\left[(1-t)^{-\frac{1}{4}}+\ln n\right] .
$$

Similarly, the second term from (34) is estimated as

$$
\begin{aligned}
& A_{42}=c(1-t)^{\frac{1}{4}} \times \\
& \times\left[\sum_{q_{2} \leqslant t_{j} \leqslant \frac{1+t}{2}}+\sum_{\frac{1+t}{2} \leqslant t_{j} \leqslant 1-n^{-2}}+\sum_{1-n^{-2} \leqslant t_{j} \leqslant 1}\right] \frac{\left|\hat{P}_{n, N}\left(t_{j}\right)\right|}{t_{j}-t} \Delta \eta_{j}= \\
& =c(1-t)^{\frac{1}{4}}\left[A_{42}^{(1)}+A_{42}^{(2)}+A_{42}^{(3)}\right] .
\end{aligned}
$$

Applying Lemma 2 and a series of transformations, we obtain estimates for these parts:

$$
A_{42}^{(1)} \leqslant c n^{\frac{1}{2}}, \quad A_{42}^{(2)} \leqslant c n^{\frac{1}{2}}, \quad A_{42}^{(3)} \leqslant c n^{\frac{1}{2}} .
$$


Then

$$
A_{4} \leqslant c\left[A_{41}+A_{42}\right] \leqslant c\left[(1-t)^{\frac{1}{4}}+\ln n\right] .
$$

Finally, all the estimates (28), (32), (33) and (40) in total allow us to display for $t \in\left[0,1-4 n^{-2}\right]$

$$
L_{n, N}(t)=A_{1}+A_{2}+A_{3}+A_{4} \leqslant c\left[(1-t)^{\frac{1}{4}}+\ln n\right] .
$$

Now we consider the behavior of the Lebesgue function $L_{n, N}(t)$ for $t \in\left[1-4 n^{-2}, 1\right]$. Let us represent the Lebesgue function in the following form:

$$
\begin{gathered}
L_{n, N}(t)=\left[\sum_{-1 \leqslant t_{j} \leqslant-\frac{1}{2}}+\sum_{-\frac{1}{2} \leqslant t_{j} \leqslant q_{1}}+\sum_{q_{1} \leqslant t_{j} \leqslant 1}\right]\left|K_{n, N}\left(t_{j}, t\right)\right| \Delta \eta_{j}= \\
=I_{1}+I_{2}+I_{3} .
\end{gathered}
$$

1. The estimation of $I_{1}$ is similar to the estimation of $A_{1}$ :

$$
\begin{gathered}
I_{1} \leqslant 2\left[\sum_{-1 \leqslant t_{j} \leqslant-1+4 n^{-2}}+\sum_{-1+4 n^{-2} \leqslant t_{j} \leqslant-\frac{1}{2}}\right]\left(\left|\hat{P}_{n+1, N}(t) \hat{P}_{n, N}\left(t_{j}\right)\right|+\right. \\
\left.+\left|\hat{P}_{n, N}(t) \hat{P}_{n+1, N}\left(t_{j}\right)\right|\right) \Delta \eta_{j}=2\left(I_{11}+I_{12}\right) .
\end{gathered}
$$

Using the weighted estimates for the discrete Legendre polynomials, we get for $I_{11}$ and $I_{12}$ the following inequalities:

$$
I_{11} \leqslant c n^{-1}, \quad I_{12} \leqslant c n^{\frac{1}{2}} .
$$

Therefore,

$$
I_{1} \leqslant c n^{\frac{1}{2}}
$$

2. To estimate $I_{2}$, we use Lemma 5 and the Christoffel - Darboux formula again:

$$
\begin{gathered}
I_{2} \leqslant C_{n, N}\left[\sum_{-\frac{1}{2} \leqslant t_{j} \leqslant 1-\frac{8}{n^{2}}} \frac{1-t_{j}}{t-t_{j}}\left|\hat{P}_{n, N}^{1,0}\left(t_{j}\right) \hat{P}_{n, N}(t)\right| \Delta \eta_{j}+\right. \\
\left.+\sum_{-\frac{1}{2} \leqslant t_{j} \leqslant 1-\frac{8}{n^{2}}} \frac{1-t}{t-t_{j}}\left|\hat{P}_{n, N}^{1,0}(t) \hat{P}_{n, N}\left(t_{j}\right)\right| \Delta \eta_{j}\right]=C_{n, N}\left[I_{21}+I_{22}\right] .
\end{gathered}
$$


Consider, firstly, $I_{21}$ :

$$
\begin{gathered}
I_{21} \leqslant c n^{\frac{1}{2}}\left[\sum_{-\frac{1}{2} \leqslant t_{j} \leqslant 0}+\sum_{0 \leqslant t_{j} \leqslant 1-\frac{8}{n^{2}}}\right] \frac{1-t_{j}}{t-t_{j}}\left|\hat{P}_{n, N}^{1,0}\left(t_{j}\right)\right| \Delta \eta_{j} \leqslant \\
\leqslant c \sum_{-\frac{1}{2} \leqslant t_{j} \leqslant 1-\frac{8}{n^{2}}} \frac{\left(1-t_{j}\right)^{\frac{1}{4}}}{t-t_{j}} \Delta \eta_{j} .
\end{gathered}
$$

Due to the obvious inequality $\left(1-t_{j}\right)^{\frac{1}{4}} \leqslant(1-t)^{\frac{1}{4}}+\left(t-t_{j}\right)^{\frac{1}{4}}$, we can rewrite

$$
\begin{aligned}
& I_{21} \leqslant c n^{\frac{1}{2}}\left[(1-t)^{\frac{1}{4}}\right.\left.\sum_{-\frac{1}{2} \leqslant t_{j} \leqslant 1-\frac{8}{n^{2}}} \frac{\Delta \eta_{j}}{t-t_{j}}+\sum_{-\frac{1}{2} \leqslant t_{j} \leqslant 1-\frac{8}{n^{2}}} \frac{\Delta \eta_{j}}{\left(t-t_{j}\right)^{\frac{3}{4}}}\right]= \\
&=c n^{\frac{1}{2}}\left[(1-t)^{\frac{1}{4}} I_{21}^{(1)}+I_{21}^{(2)}\right] .
\end{aligned}
$$

Using Lemma 1, we obtain for these new parts the estimates

$$
I_{21}^{(1)} \leqslant c \ln n, \quad I_{21}^{(2)} \leqslant c,
$$

and finally

$$
I_{21} \leqslant c n^{\frac{1}{2}}\left(n^{-\frac{1}{2}} \ln n+1\right) \leqslant c n^{\frac{1}{2}} .
$$

Let us start with $I_{22}$. Using the weighted estimates and the fact that $\left(1+t_{j}\right)^{-\frac{1}{4}} \leqslant 3^{\frac{1}{4}}\left(1-t_{j}\right)^{-\frac{1}{4}} \leqslant c n^{\frac{1}{2}}$ for $-\frac{1}{2} \leqslant t_{j} \leqslant 0$, we derive

$$
\begin{gathered}
I_{22} \leqslant c n^{-\frac{1}{2}} \sum_{-\frac{1}{2} \leqslant t_{j} \leqslant 1-\frac{8}{n^{2}}} \frac{\left(1-t_{j}\right)^{-\frac{1}{4}}}{t-t_{j}} \Delta \eta_{j} \leqslant \\
\leqslant c n^{-\frac{1}{2}} n^{\frac{1}{2}} \sum_{-\frac{1}{2} \leqslant t_{j} \leqslant 1-\frac{8}{n^{2}}} \frac{\Delta \eta_{j}}{t-t_{j}} \leqslant c \ln n .
\end{gathered}
$$

Combining (45) and (46), we finally get

$$
I_{2} \leqslant C_{n, N}\left[I_{21}+I_{22}\right] \leqslant c\left(n^{\frac{1}{2}}+\ln n\right) \leqslant c n^{\frac{1}{2}} .
$$

3. For the last part, we just use (24):

$$
I_{3} \leqslant \sum_{1-n^{-2} \leqslant t_{j} \leqslant 1} \sum_{k=0}^{n}\left|\hat{P}_{k, N}(t) \hat{P}_{k, N}\left(t_{j}\right)\right| \Delta \eta_{j} \leqslant
$$




$$
\leqslant c \sum_{1-n^{-2} \leqslant t_{j} \leqslant 1} \sum_{k=0}^{n} k^{\frac{1}{2}} k^{\frac{1}{2}} \Delta \eta_{j} \leqslant c n^{2} \sum_{1-n^{-2} \leqslant t_{j} \leqslant 1} \Delta \eta_{j} \leqslant c(a) .
$$

Combining estimates (43), (47) and (48), we finally get

$$
L_{n, N}(t)=I_{1}+I_{2}+I_{3} \leqslant c\left(n^{\frac{1}{2}}+\ln n\right), \quad t \in\left[1-4 n^{-2}, 1\right] .
$$

Note that for $t \in\left[1-4 n^{-2}, 1\right]$ the expressions $\left(1-t^{2}\right)^{\frac{1}{4}}$ and $n^{\frac{1}{2}}$ are of the same order. Hence, from (41) and (49) we deduce the assertion of the theorem.

Returning to (25), we also get the following statement from Theorem 1:

Theorem 2. The estimate

$$
\left|R_{n, N}(t)\right| \leqslant c(\gamma) E_{n}(f)\left[\ln n+\left(\sqrt{1-t^{2}}+\frac{1}{n}\right)^{-\frac{1}{2}}\right]
$$

holds for the remainder $R_{n, N}(t)$, where $2 \leqslant n \leqslant \gamma \lambda_{N}^{-1 / 3}, \gamma>0$, and $E_{n}(f)$ is the best approximation for the function $f(t)$ by polynomials of degree at most $n$.

6. Some applications. Once again, let $f(t)$ be a continuous on $[-1,1]$ function, which is measured at the nodes of some arbitrary grid $\Omega_{N}=\left\{t_{j}\right\}_{j=0}^{N-1}$, satisfying (1)-(2). We denote these measurements by $y_{j}=f\left(t_{j}\right)+\xi_{j}, 0 \leqslant j \leqslant N-1$. Here $\xi_{j}$ are observation errors, which are independent random variables satisfying the following conditions:

$$
E\left[\xi_{j}\right]=0, \quad E\left[\xi_{i} \xi_{j}\right]=\sigma_{j}^{2} \delta_{i j}, \quad 0 \leqslant j \leqslant N-1,
$$

where $E[X]$ is the expected value of a random variable $X$. It is required to approximately restore $f(t)$ at the point $t \in[-1,1]$ using discrete information $\left\{y_{j}\right\}_{j=0}^{N-1}$. To solve this problem, we introduce an algebraic polynomial $S_{n, N}(t)$ that minimizes the sum

$$
J\left(a_{0}, \ldots, a_{n}\right)=\sum_{j=0}^{N-1}\left(y_{j}-p_{n}\left(t_{j}\right)\right)^{2} \rho_{j}
$$

on the set of all polynomials $p_{n}(t)=a_{0}+a_{1} t+\ldots+a_{n} t^{n}$ of degree $n \leqslant N-1$, where $\rho_{j}$ are positive weight factors. 
The question is, how precise $S_{n, N}(t)$ approximates the original function $f(t)$ at $t \in[-1,1]$, i. e., it is required to estimate the value $\left(f(t)-S_{n, N}(t)\right)^{2}$. Since this value depends on random errors $\xi_{0}, \ldots, \xi_{N-1}$, a more accurate formulation of the problem is to estimate its average value

$$
J_{n, N}(f, t)=E\left[\left(f(t)-S_{n, N}(t)\right)^{2}\right] .
$$

In [1] this problem was studied for the uniform grid $t_{j}=-1+\frac{2 j}{N-1}$, $\rho_{j}=1,0 \leqslant j \leqslant N-1$. In this article, we consider a more general case when the nodes $t_{j}$ form a non-uniform grid $\Omega_{N}=\left\{t_{j}\right\}_{j=0}^{N-1} \subset[-1,1]$, and weights $\rho_{j}$ satisfy certain natural conditions.

More precisely, the values of $\sigma_{j}$, appearing in (50), and corresponding weights $\rho_{j}$ are defined for a given real $\sigma$ using equalities

$$
\sigma_{j}^{2}=\sigma^{2} \frac{\lambda_{N}}{\Delta \eta_{j}}, \quad \rho_{j}=\left(\sigma / \sigma_{j}\right)^{2}=\frac{\Delta \eta_{j}}{\lambda_{N}}
$$

It is well-known (see [15]) that polynomials $S_{n, N}(t)$ minimizing the value (51), can be represented as

$$
S_{n, N}(t)=\sum_{k=0}^{n} \hat{y}_{k} \hat{P}_{k, N}(t), \quad \text { where } \quad \hat{y}_{k}=\sum_{j=0}^{N-1} y_{j} \hat{P}_{k, N}\left(t_{j}\right) \Delta \eta_{j} .
$$

Let $\Lambda_{n, N}(f, t)$ be the partial Fourier sum of order $n$ for the original (noiseless) function $f=f(t)$ by the system $\left\{\hat{P}_{k, N}\right\}_{k=0}^{N-1}$, i. e.,

$$
\Lambda_{n, N}(f, t)=\sum_{k=0}^{n} \hat{f}_{k} \hat{P}_{k, N}(t), \text { where } \hat{f}_{k}=\sum_{j=0}^{N-1} f\left(t_{j}\right) \hat{P}_{k, N}\left(t_{j}\right) \Delta \eta_{j} .
$$

From (50) it follows that $\Lambda_{n, N}(f, t)=E\left[S_{n, N}(t)\right]$. In fact,

$$
E\left[S_{n, N}(t)\right]=E\left[\sum_{k=0}^{n} \hat{y}_{k} \hat{P}_{k, N}(t)\right]=\sum_{k=0}^{n} E\left[\hat{y}_{k}\right] \hat{P}_{k, N}(t),
$$

where

$$
E\left[\hat{y}_{k}\right]=E\left[\sum_{j=0}^{N-1}\left(f\left(t_{j}\right)+\xi_{j}\right) \hat{P}_{k, N}\left(t_{j}\right) \Delta \eta_{j}\right]=
$$




$$
=\sum_{j=0}^{N-1} E\left[f\left(t_{j}\right)+\xi_{j}\right] \hat{P}_{k, N}\left(t_{j}\right) \Delta \eta_{j}=\hat{f}_{k} .
$$

In addition, it can be shown that

$$
\begin{aligned}
J_{n, N}(f, t)=(f(t) & \left.-\Lambda_{n, N}(f, t)\right)^{2}+\sigma^{2} \lambda_{N} \sum_{k=0}^{n}\left(\hat{P}_{k, N}(t)\right)^{2}= \\
= & R_{n, N}^{2}(f, t)+D_{n, N}(t) .
\end{aligned}
$$

To do this, consider the expression

$$
\begin{gathered}
\Delta_{n, N}(f, t)=S_{n, N}(f, t)-\Lambda_{n, N}(f, t)=\sum_{k=0}^{n}\left(\hat{y}_{k}-\hat{f}_{k}\right) \hat{P}_{k, N}(t)= \\
=\sum_{k=0}^{n} \hat{\xi}_{k} \hat{P}_{k, N}(t)=\sum_{k=0}^{n} \sum_{j=0}^{N-1} \xi_{j} \hat{P}_{k, N}(t) \hat{P}_{k, N}\left(t_{j}\right) \Delta \eta_{j} .
\end{gathered}
$$

We will need the expected value of this value:

$$
\begin{aligned}
& E\left[\Delta_{n, N}(f, t)\right]=\sum_{k=0}^{n} E\left[\hat{\xi}_{k}\right] \hat{P}_{k, N}(t)= \\
= & \sum_{k=0}^{n} \sum_{j=0}^{N-1} E\left[\xi_{j}\right] \hat{P}_{k, N}(t) \hat{P}_{k, N}\left(t_{j}\right) \Delta \eta_{j}=0,
\end{aligned}
$$

and its square:

$$
\begin{gathered}
E\left[\Delta_{n, N}^{2}(f, t)\right]=E\left[\left(\sum_{k=0}^{n} \hat{\xi}_{k} \hat{P}_{k, N}(t)\right)\left(\sum_{l=0}^{n} \hat{\xi}_{l} \hat{P}_{l, N}(t)\right)\right]= \\
=\sum_{k=0}^{n} \sum_{l=0}^{n} E\left[\hat{\xi}_{k} \hat{\xi}_{l}\right] \hat{P}_{k, N}(t) \hat{P}_{l, N}(t) .
\end{gathered}
$$

Due to (50) and (4), we know that

$$
E\left[\hat{\xi}_{k} \hat{\xi}_{l}\right]=\sum_{i=0}^{N-1} \sum_{j=0}^{N-1} E\left[\xi_{i} \xi_{j}\right] \hat{P}_{k, N}\left(t_{i}\right) \hat{P}_{l, N}\left(t_{j}\right) \Delta \eta_{i} \Delta \eta_{j}=
$$




$$
=\sum_{j=0}^{N-1}\left(\sigma_{j}^{2} \Delta \eta_{j}\right) \hat{P}_{k, N}\left(t_{j}\right) \hat{P}_{l, N}\left(t_{j}\right) \Delta \eta_{j}=\sigma^{2} \lambda_{N} \delta_{k l}
$$

wherefrom

$$
E\left[\Delta_{n, N}^{2}(f, t)\right]=\sigma^{2} \lambda_{N} \sum_{k=0}^{n}\left(\hat{P}_{k, N}(t)\right)^{2} .
$$

Then, taking into account (54) and (55), we have

$$
\begin{gathered}
J_{n, N}(f, t)=E\left[\left(f(t)-S_{n, N}(f, t)\right)^{2}\right]= \\
=E\left[\left(f(t)-\Lambda_{n, N}(f, t)+\Delta_{n, N}(f, t)\right)^{2}\right]=\left(f(t)-\Lambda_{n, N}(f, t)\right)^{2}+ \\
+2\left(f(t)-\Lambda_{n, N}(f, t)\right) E\left[\Delta_{n, N}(f, t)\right]+E\left[\Delta_{n, N}^{2}(f, t)\right]= \\
=\left(f(t)-\Lambda_{n, N}(f, t)\right)^{2}+\sigma^{2} \lambda_{N} \sum_{k=0}^{n}\left(\hat{P}_{k, N}(t)\right)^{2}=R_{n, N}^{2}(f, t)+D_{n, N}(t) .
\end{gathered}
$$

Thus, the original objective of estimating the deviation of partial sums by discrete Legendre polynomials $\hat{P}_{n, N}(t)$ from the desired function $f(t)$ comes to estimating these two values: $R_{n, N}^{2}(f, t)$ and $D_{n, N}(t)$.

The estimate for $R_{n, N}(f, t)$ is given in Theorem 2. Let us consider the value $D_{n, N}(t)=\sigma^{2} \lambda_{N} \sum_{k=0}^{n}\left(\hat{P}_{k, N}(t)\right)^{2}$. Using weighted estimates (24) obtained in Theorem A, we have

$$
\begin{aligned}
& D_{n, N}(t) \leqslant \sigma^{2} \lambda_{N} \sum_{k=0}^{n}\left(c(a)\left(1+B \sqrt{k^{3} \lambda_{N}}\right) k^{\frac{1}{2}}\right)^{2} \leqslant \\
& \leqslant c(a) \sigma^{2}\left(n^{2} \lambda_{N}\right)\left(1+B \sqrt{n^{3} \lambda_{N}}\right)^{2} \leqslant c(a) \sigma^{2}\left(n^{\frac{5}{2}} \lambda_{N}\right)^{2} .
\end{aligned}
$$

So, the value $D_{n, N}(t)$ tends to zero when $n=O\left(\lambda_{N}^{-1 / 3}\right)$.

Finally, we conclude with the following statement.

Theorem 3. Let $f(t)$ be a continuous on $[-1,1]$ function given by its measurements $y_{j}=f\left(t_{j}\right)+\xi_{j}, j=0,1, \ldots, N-1$, in the nodes of the grid $\Omega_{N}$, which satisfy (1)-(2), where $\xi_{j}$ are independent random mistakes of observation that satisfy (50)-(52). Then, for $2 \leqslant n \leqslant \gamma \lambda_{N}^{-1 / 3}, \gamma>0$, the following estimate holds:

$$
J_{n, N}(f, t) \leqslant c(a, \gamma, \sigma)\left(E_{n}(f)\left[\ln n+\left(\sqrt{1-t^{2}}+\frac{1}{n}\right)^{-\frac{1}{2}}\right]+\lambda_{N}^{\frac{1}{3}}\right),
$$


where $E_{n}(f)$ is the best approximation for the function $f(t)$ by polynomials of degree at most $n$.

\section{References}

[1] Sharapudinov I. I. Convergence of the method of least squares. Math. Notes, 1993, vol. 53, no. 3, pp. 335-344.

DOI: https://doi.org/10.1007/BF01207722

[2] Nurmagomedov A. A. Mnogochleny, ortogonal'niye na neravnomernih setkah (Polynomials, orthogonal on non-uniform grids). Izv. Sarat. Univ. (N. S.) Ser. Mat. Mekh. Inform., 2011, vol. 11, no. 3, part 2, pp. 29-42 (in Russian).

[3] Nurmagomedov A. A. Convergence of Fourier sums in polynomials orthogonal on arbitrary grids. Russian Math. (Iz. VUZ), 2012, vol. 56, no. 7, pp. 52-54 (in Russian).

DOI: https://doi.org/10.3103/S1066369X12070080

[4] Szego G. Orthogonal Polynomials. AMS Colloq. Publ, 1939, vol. 23.

DOI: https://doi.org/10.1090/coll/023

[5] Bari N. K. Obobsheniye neravenstv S.N. Bernshteina i A.A. Markova (Generalization of inequalities of S.N. Bernshtein and A.A. Markov). Izv. AS USSR. Ser. matem., 1954, vol. 18:2, pp. 159-176 (in Russian).

[6] Konyagin S. V. Markov's inequality for polynomials in the metric of $L$. Proc. Steklov Inst. Math., 1981, vol. 145, pp. 129-138.

[7] Sharapudinov I. I. Mixed series by classical orthogonal polynomials. Daghestan Electronic Mathematical Reports, 2015, vol. 3, pp. 1-254 (in Russian).

DOI: https://doi.org/10.31029/demr.3.1

[8] Sharapudinov I. I. Asimptotika polinomov, ortogonal'nykh na setkakh iz edinichnoi okruzhnosti $i$ chislovoi priamoi (Asymptotics of polynomials, orthogonal on nets from unite circle and number line). Sovremennye problemy matematiki, mekhaniki, informatiki (Modern problems of mathematics, mechanics, informatics). Materials of the international scientific conference (Tula, November, 23-27), 2009, pp. 100-106 (in Russian).

[9] Sharapudinov I. I. Nekotorye svoistva polinomov, ortogonal'nykh na neravnomernykh setkakh iz edinichnoi okruzhnosti i otrezka (On some properties of polynomials orthogonal on non-uniform grids from unite circle and number line). Sovremennye problemy teorii funktsii i ikh prilozheniia (Modern problems of function theory and its applications). Materials of the international scientific conference "15-th Saratov winter school, dedicated 
to the 125th anniversary of V. V. Golubev and 100th anniversary of SGU" (Saratov, January, 28 - February, 3), 2010, p. 187 (in Russian).

[10] Sharapudinov I. I. Asimptoticheskie svoistva polinomov, ortogonal'nykh na konechnykh setkakh edinichnoi okruzhnosti (Asymptotic properties of the polynomials orthogonal on the finite nets of the unite circle). Herald of Daghestan Scientific Center of RAS, 2011, vol. 42, pp. 5-14 (in Russian).

[11] Nurmagomedov A. A. Ob asimptotike mnogochlenov, ortogonal'nykh na proizvol'nykh setkakh (About approximation polynomials, orthogonal on random grids). Izv. Sarat. Univ. (N.S.) Ser. Mat. Mekh. Inform., 2008, vol. 8, no. 1, pp. 25-31 (in Russian).

[12] Nurmagomedov A.A. Asimptoticheskie svoistva mnogochlenov $\hat{p}_{n}^{\alpha, \beta}(x)$, ortogonal'nykh na proizvol'nykh setkakh $v$ sluchae tselykh $\alpha$ and $\beta$ (Asymptotic properties of polynomials $\hat{p}_{n}^{\alpha, \beta}(x)$, orthogonal on any sets in the case of integers $\alpha$ and $\beta$ ). Izv. Sarat. Univ. (N.S.) Ser. Mat. Mekh. Inform., 2010, vol. 10, no. 2, pp. 10-19 (in Russian).

[13] Sultanakhmedov M.S. Asimptoticheskie svoistva i vesoviye otsenki polinomov, ortogonal'nykh na neravnomernoy setke s vesom Yakobi (Asymptotic Properties and Weighted Estimation of Polynomials, Orthogonal on the Nonuniform Grids with Jacobi Weight). Izv. Sarat. Univ. (N.S.) Ser. Mat. Mekh. Inform., 2014, vol. 14, no. 1, pp. 38-46 (in Russian).

DOI: https://doi.org/10.18500/1816-9791-2014-14-1-38-47

[14] Alexits G. Konvergenzprobleme der Orthogonalreihen (Convergence problems of orthogonal series). Veb Deutscher Verlag der Wissenschaften, Berlin, 1960. DOI: https://doi.org/10.1002/zamm.19620420423

[15] Chebyshev P.L. Polnoe sobraniye sochineniy (Full composition of writings). M.: Izd-vo AN SSSR, vol. 2-3, 1947-1951 (in Russian).

Received June 3, 2019.

In revised form, October 18, 2019.

Accepted October 22, 2019.

Published online November 9, 2019.

Dagestan Scientific Center of RAS

45, M.Gadzhieva st., Makhachkala, 367025, Russia

E-mail: sultanakhmedov@gmail.com 